\title{
Analysis of lymph node metastasis in early gastric cancer: a single institutional experience from China
}

\author{
Jinggui Chen ${ }^{1,2}$, Guangfa Zhao ${ }^{1,2}$ and Yanong Wang ${ }^{1,2^{*}}$
}

\begin{abstract}
Background: Lymph node metastasis (LNM) has a strong influence on the prognosis of patients with early gastric cancer (EGC). The aim of this study was to reveal the incidence of LNM and evaluate risk factors for LNM to determine the appropriate treatment for EGC in a Chinese population.

Methods: Patients who underwent radical gastrectomy with lymph node dissection for EGC between 2012 and 2017 were retrospectively analyzed. Univariate and multivariate analyses were conducted to identify clinicopathological features that were risk factors for LNM.

Results: A total of 1033 patients with EGC were enrolled. Of these patients, 668 (64.7\%) were men, and 365 (35.3\%) were women, ranging in age from 19 to 82 years (mean $56.9 \pm 10.9$ years). LNM was detected in 173(16.7\%) patients with EGC. Among 508 patients with mucosal cancer, 44 (8.7\%) patients had LNM. In 525 patients with submucosal cancer, the incidence of LNM was 24.6\% (129/525). The age, gender, tumor size, type of differentiation, Lauren classification, and lymphovascular and perineural invasion showed a significant correlation with the rate of LNM in EGC by univariate and multivariate analyses. Patients with submucosal gastric cancer had an older age, a higher proportion of proximal lesion, larger tumor size, more frequent lymphovascular invasion, perineural invasion, and more LNM than patients with mucosal gastric cancer.
\end{abstract}

Conclusions: Our study revealed a relatively high incidence of LNM in EGC, compared with Japanese and Korean cohorts. Female sex, large tumor size, undifferentiated-type, and lymphovascular invasion were independent risk factors for LNM in EGC. Radical gastrectomy with lymphadenectomy should be performed in EGC patients with a high risk of LNM.

Keywords: Early gastric cancer, Lymph node metastasis, Risk factors

\section{Introduction}

Early gastric cancer (EGC), which is defined as tumor confined to the mucosa or submucosa, regardless of the presence or absence of lymph node metastasis (LNM), has a better prognosis than advanced gastric cancer [1]. LNM is deemed as the most important prognostic factor

\footnotetext{
* Correspondence: wyfuscc@163.com

'Department of Gastric Surgery, Fudan University Shanghai Cancer Center, No. 270 Dongan Road, Shanghai 200032, People's Republic of China ${ }^{2}$ Department of Oncology, Shanghai Medical College, Fudan University, No. 270 Dongan Road, Shanghai 200032, People's Republic of China
}

[2, 3]. Previous study reported that the 5-year survival rate was $87.3 \%$ in EGC patients with LNM and $94.2 \%$ in patients without LNM, respectively [4]. The incidence of LNM in EGC varies obviously in EGC patients with different depth of invasion. The incidence of LNM in EGC with submucosal invasion is significantly higher than which with mucosal confined (8-25\% vs $2-5 \%$ ) [5-9].

Nowadays, endoscopic resection (ER) has been widely accepted as an alternative curative procedure for EGC patients without LNM $[10,11]$. According to the Japanese 
gastric cancer treatment guidelines, ER is indicated as a standard treatment for a differentiated-type EGC, of which the depth of invasion is clinically diagnosed as T1a, no ulcerative finding exists, and the tumor size is no more than $2.0 \mathrm{~cm}$ [12]. Therefore, it is important to precisely identify those patients with high risk for LNM when deciding the optimal treatment strategy for patients with EGC.

Gastric cancer is the second leading cause of cancerrelated death in China, with an estimated more than sixty thousand new cases diagnosed each year [13]. However, the proportion of EGC only accounts about $20 \%$ for patients with gastric cancer, and the majority of patients are diagnosed with advanced gastric cancer [13], resulting in a relatively low survival rate of gastric cancer in China compared with Japan and Korea. The present study involved a large number of EGC patients in our institution and retrospectively investigated the incidence of LNM of EGC and the relationship between the clinicopathologic factors and LNM. We aim to identify the risk factors for LNM in EGC patients and provide the basis for further research on individual management of $\mathrm{EGC}$ in China.

\section{Patients and method}

\section{Patients}

We consecutively enrolled 1033 patients with EGC who underwent curative gastrectomy with regional lymphadenectomy between 2012 and 2017 at the Department of Gastric Surgery, Fudan University Shanghai Cancer Center, and retrospectively reviewed their medical records. Endoscopic resection was not practiced routinely in our institution, and all the resectable gastric cancer received radical gastrectomy with lymphadenectomy. Patients underwent subtotal or total gastrectomy depending on tumor location, with D1+ or D2 lymphadenectomy in accordance with the guidelines of the Japanese Research Society for Gastric Cancer. We excluded patients with multiple lesions or preoperative treatment history. None of the patients received endoscopic treatment, neoadjuvant chemotherapy, or radiotherapy. Relationships between clinicopathological characteristics and LNM were examined to identify the risk factors. These clinicopathological parameters were analyzed: sex, age, location of tumor, size of tumor, depth of invasion, lymphovascular invasion (LVI), perineural invasion, Lauren classification, and type of differentiation. The ethical committee of Fudan University Shanghai Cancer Center approved this study. The study was performed in accordance with the Declaration of Helsinki and its revisions.

\section{Histopathologic evaluation}

The primary gastric adenocarcinoma and retrieved LN were routinely examined and reviewed by experienced pathologists. Differentiated-type EGC included papillary adenocarcinoma, well or moderately differentiated adenocarcinoma. Poorly or undifferentiated adenocarcinoma and signet ring cell carcinoma were classified as undifferentiated-type EGC. EGC was divided into mucosal and submucosal gastric cancer according to the depth of invasion. Tumor size was recorded as the maximum diameter of the tumor. The analyses of this study were based on postoperative pathology of resected specimens. The pathological staging was performed according to the American Joint Committee on Cancer 7th edition.

\section{Statistical analysis}

The statistical analyses were done using the SPSS software (version 22.0, IBM Inc., Armonk, NY). The risk factors for LNM were identified by performing a Student $t$ test, chi-squared test, or Fisher's exact test. Multivariate logistic regression analysis was used to identify the independent variables associated with LNM. A $P$ value of less than 0.05 was considered to be statistically significant.

\section{Results \\ Patient characteristics}

A total of 1033 patients with EGC were studied. Of these patients, $668(64.7 \%)$ were men, and 365 (35.3\%) were women, ranging in age from 19 to 82 years (mean $56.9 \pm$ 10.9 years). LNM was detected in 173 (16.7\%) patients with EGC. Among 508 patients with tumor confined to the mucosa, 44 (8.7\%) patients had LNM. In 525 patients with tumor invasion to the submucosa, the incidence of LNM was $24.6 \%$ (129 out of 525). One hundred-five (10.2\%) of EGC were located in the upper third of the stomach, and 204 (19.7\%) were located in the middle, and $724(70.1 \%)$ were located in the lower third. The mean diameter of the tumor for EGC was $2.1 \pm 1.2 \mathrm{~cm}$. The mean number of retrieved LNs was $24.1 \pm 8.2$.

\section{Risk factors for LNM in EGC}

The association between LNM and various clinicopathological factors was examined by univariate analysis (Table 1). There were no significant differences between patients with and without LNM in terms of tumor location and number of retrieved LNs. The age, sex, tumor size, invasion depth, lymphovascular invasion (LVI), tumor differentiation, perineural invasion, and Lauren classification showed an association with LNM. Patients with LNM were younger than those without LNM (54.2 \pm 11.3 vs $57.4 \pm 10.8, P<0.001)$. The proportion of patients with LNM was higher in female patients than male patients $(24.1 \%$ vs $12.7 \%, P<0.001)$. A greater tumor size $(2.5 \pm 1.3 \mathrm{~cm}$ vs $2.1 \pm 1.1, P<0.001)$ and LVI positive $(55.4 \%$ vs $12.6 \%, P<0.001)$ were related with more frequent LNM. In the patients with undifferentiated-type cancer, the rate of LNM was much higher than the 
Table 1 Univariate analysis of risk factors for lymph node metastasis in early gastric cancer

\begin{tabular}{|c|c|c|c|}
\hline Variables & LN negative $(n=860)$ & LN positive $(n=173)$ & $P$ \\
\hline Age, years & $57.4 \pm 10.8$ & $54.2 \pm 11.3$ & $<0.001$ \\
\hline \multicolumn{4}{|l|}{ Sex, $n(\%)$} \\
\hline Male & $583(87.3)$ & $85(12.7)$ & \multirow[t]{2}{*}{$<0.001$} \\
\hline Female & $277(75.9)$ & $88(24.1)$ & \\
\hline \multicolumn{4}{|l|}{ Location, $n$ (\%) } \\
\hline Upper & $93(85.6)$ & $12(11.4)$ & \multirow[t]{3}{*}{0.262} \\
\hline Middle & $166(81.4)$ & $38(18.6)$ & \\
\hline Lower & $601(83.0)$ & $123(17.0)$ & \\
\hline Tumor size, cm & $2.1 \pm 1.1$ & $2.5 \pm 1.3$ & $<0.001$ \\
\hline Retrieved LN & $23.9 \pm 7.9$ & $25.1 \pm 9.3$ & 0.062 \\
\hline \multicolumn{4}{|l|}{ Invasion depth } \\
\hline Mucosal & $464(91.3)$ & $44(8.7)$ & \multirow[t]{2}{*}{$<0.001$} \\
\hline Submucosal & $396(75.4)$ & $129(24.6)$ & \\
\hline \multicolumn{4}{|c|}{ Lymphovascular invasion, $n$ (\%) } \\
\hline Negative & $815(87.4)$ & $117(12.6)$ & \multirow[t]{2}{*}{$<0.001$} \\
\hline Positive & $45(44.6)$ & $56(55.4)$ & \\
\hline \multicolumn{4}{|c|}{ Perineural invasion, $n$ (\%) } \\
\hline Negative & $846(84.5)$ & $155(15.5)$ & \multirow[t]{2}{*}{$<0.001$} \\
\hline Positive & $14(43.7)$ & $18(56.3)$ & \\
\hline \multicolumn{4}{|c|}{ Differentiation, $n(\%)$} \\
\hline D-type & $397(91.5)$ & $37(8.5)$ & \multirow[t]{2}{*}{$<0.001$} \\
\hline UD-type & $463(77.3)$ & $136(22.7)$ & \\
\hline \multicolumn{4}{|c|}{ Lauren classification ${ }^{a}, n(\%)$} \\
\hline Intestinal & $427(89.9)$ & $48(10.1)$ & \multirow[t]{3}{*}{$<0.001$} \\
\hline Diffuse & $218(76.8)$ & $66(23.2)$ & \\
\hline Mixed & $127(74.7)$ & $43(25.3)$ & \\
\hline
\end{tabular}

D-type differentiated-type, $L N$ lymph node, UD-type undifferentiated-type

${ }^{a}$ There are some patients with unknown information about the

Lauren classification

differentiated-type $(22.7 \%$ vs $8.5 \%, P<0.001)$. Perineural invasion was also related to an increased risk of LNM (56.3\% vs $15.5 \%, P<0.001)$. The diffuse or mixed histologic type showed a more frequent LNM than the intestinal type $(P<0.001)$. Multivariate analysis further showed that age, sex, tumor size, invasion depth, type of differentiation, LVI, and perineural invasion had significant influences on LNM in EGC (Table 2).

\section{Clinicopathologic characteristics of mucosal cancer compared with submucosal cancer}

Table 3 shows the clinicopathologic characteristics of mucosal and submucosal cancers. Age, tumor location, size of the tumor, LNM status, LVI, perineural invasion, and Lauren classification and LN differed significantly between the 2 groups. Patients with submucosal cancer had older age $(57.9 \pm 10.4$ vs $55.8 \pm 11.3, P=0.002)$, a
Table 2 Multivariate analysis of risk factors for lymph node metastasis in early gastric cancer

\begin{tabular}{llll}
\hline Variables & Odds ratio & 95\% confidence interval & $P$ \\
\hline Age & 0.976 & $0.959-0.992$ & 0.004 \\
Male sex & 0.47 & $0.321-0.688$ & $<0.001$ \\
Tumor size & 1.272 & $1.095-1.485$ & 0.002 \\
Submucosal invasion & 2.781 & $1.832-4.221$ & $<0.001$ \\
Undifferentiated-type & 2.103 & $1.362-3.247$ & 0.001 \\
Lymphovascular invasion & 5.383 & $3.318-8.732$ & $<0.001$ \\
Perineural invasion & 3.61 & $1.560-8.306$ & 0.003 \\
\hline
\end{tabular}

Table 3 Clinicopathologic characteristics of mucosal gastric cancer compared with submucosal gastric cancer

\begin{tabular}{llll}
\hline Variables & Mucosal $(n=508)$ & Submucosal $(n=525)$ & $P$ \\
\hline $\begin{array}{l}\text { Age, years } \\
\text { Sex, } n(\%)\end{array}$ & $55.8 \pm 11.3$ & $57.9 \pm 10.4$ & 0.002 \\
$\quad$ Male & $314(61.8)$ & $354(67.4)$ & 0.059 \\
Female & $194(38.2)$ & $171(32.6)$ & \\
Location, $n(\%)$ & & & 0.011 \\
$\quad$ Upper & $37(7.3)$ & $68(13.0)$ & \\
Middle & $104(20.5)$ & $100(19.0)$ & $<0.001$ \\
Lower & $367(72.2)$ & $357(68.0)$ & 0.100 \\
Tumor size, cm & $2.0 \pm 1.2$ & $2.3 \pm 1.1$ & $<0.001$ \\
Retrieved LN & $24.5 \pm 8.2$ & $23.7 \pm 8.1$ & \\
LN metastasis, $n(\%)$ & & $<0.001$ \\
Negative & $464(91.3)$ & $396(75.4)$ & \\
Positive & $44(8.7)$ & $129(24.6)$ & \\
Lymphovascular invasion, $n(\%)$ & & $439(83.6)$ & \\
Negative & $493(97.0)$ & $86(16.4)$ &
\end{tabular}

Perineural invasion, $n(\%)$

$\begin{array}{llll}\text { Negative } & 508(100.0) & 493(93.9) & <0.001 \\ \text { Positive } & 0(0.0) & 32(6.1) & \end{array}$

Differentiation, $n(\%)$

\begin{tabular}{llll} 
D-type & $217(42.7)$ & $217(41.3)$ & 0.653 \\
UD-type & $291(57.3)$ & $308(58.7)$ & \\
Lauren classification ${ }^{\mathrm{a}}, n(\%)$ & & 0.003 \\
Intestinal & $213(47.1)$ & $262(54.9)$ & \\
Diffuse & $77(17.0)$ & $93(19.5)$ & \\
Mixed & $162(35.9)$ & $122(25.6)$ & \\
\hline
\end{tabular}

$D$-type differentiated-type, $L N$ lymph node, UD-type undifferentiated-type

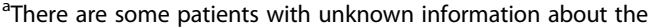
Lauren classification 
Table 4 Risk factors for lymph node metastasis in mucosal and submucosal gastric cancer

\begin{tabular}{|c|c|c|c|c|c|c|}
\hline \multirow[t]{2}{*}{ Variables } & \multicolumn{3}{|c|}{ Mucosal gastric cancer } & \multicolumn{3}{|c|}{ Submucosal gastric cancer } \\
\hline & $\mathrm{LN}(-)(N=464)$ & $\mathrm{LN}(+)(N=44)$ & $P$ & $\operatorname{LN}(-)(N=396)$ & $\mathrm{LN}(+)(N=129)$ & $P$ \\
\hline Age, years & $56.4 \pm 11.2$ & $49.8 \pm 11.6$ & $<0.001$ & $58.7 \pm 10.2$ & $55.7 \pm 10.8$ & 0.005 \\
\hline Sex, $n(\%)$ & & & $<0.001$ & & & 0.001 \\
\hline Male & $301(95.9)$ & $13(4.1)$ & & $282(79.7)$ & $72(20.3)$ & \\
\hline Female & $163(84.0)$ & $31(16.0)$ & & $114(66.7)$ & $57(33.3)$ & \\
\hline Location, $n(\%)$ & & & 0.25 & & & 0.226 \\
\hline Upper & $36(97.3)$ & $1(2.7)$ & & $57(83.8)$ & $11(16.2)$ & \\
\hline Middle & $92(88.5)$ & $12(11.5)$ & & $74(84.0)$ & $26(26.0)$ & \\
\hline Lower & $336(91.6)$ & $31(8.4)$ & & $265(74.2)$ & $92(25.8)$ & \\
\hline Tumor size, cm & $1.9 \pm 1.1$ & $2.6 \pm 1.4$ & $<0.001$ & $2.2 \pm 1.1$ & $2.5 \pm 1.2$ & 0.007 \\
\hline Retrieved LN & $24.3 \pm 8.1$ & $26.3 \pm 9.0$ & 0.126 & $23.3 \pm 7.6$ & $24.7 \pm 9.5$ & 0.085 \\
\hline Lymphovascular invasion, $n$ (\%) & & & $<0.001$ & & & $<0.001$ \\
\hline Negative & $456(92.5)$ & $37(7.5)$ & & $359(81.8)$ & $80(18.2)$ & \\
\hline Positive & $8(53.3)$ & $7(46.7)$ & & $37(43.0)$ & $49(57.0)$ & \\
\hline Perineural invasion, $n$ (\%) & & & NA & & & $<0.001$ \\
\hline Negative & $464(91.3)$ & $44(8.7)$ & & $382(77.5)$ & $111(22.5)$ & \\
\hline Positive & $0(0.0)$ & $0(0.0)$ & & $14(43.8)$ & $18(56.2)$ & \\
\hline Differentiation, $n$ (\%) & & & & & & $<0.001$ \\
\hline D-type & $214(98.6)$ & $3(1.4)$ & $<0.001$ & $183(84.3)$ & $34(15.7)$ & \\
\hline UD-type & $250(85.9)$ & $41(14.1)$ & & $213(69.2)$ & $95(30.8)$ & \\
\hline Lauren classification ${ }^{\mathrm{a}}, n(\%)$ & & & $<0.001$ & & & $<0.001$ \\
\hline Intestinal & $207(97.1)$ & $6(2.9)$ & & $220(84.0)$ & $42(16.0)$ & \\
\hline Diffuse & $139(85.7)$ & $23(14.3)$ & & $79(64.8)$ & $43(35.2)$ & \\
\hline Mixed & $68(88.3)$ & $9(11.7)$ & & $59(63.4)$ & $34(36.6)$ & \\
\hline
\end{tabular}

$D$-type differentiated-type, $L N$ lymph node, $N A$ not available, UD-type undifferentiated-type

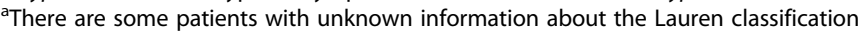

higher proportion of proximal lesion $(13.0 \%$ vs $7.3 \%, P=$ $0.011)$, larger size $(2.3 \pm 1.1 \mathrm{~cm}$ vs $2.0 \pm 1.2 \mathrm{~cm}, P<0.001)$, more frequent LVI, perineural invasion $(16.4 \%$ vs $3.0 \%, P$ $<0.001 ; 6.1 \%$ vs $0.0 \%, P<0.001$, respectively), and more LNM $(24.6 \%$ vs $8.7 \%, P<0.001)$ than patients with mucosal cancer. In terms of Lauren classification, the diffuse or mixed histologic type was observed significantly more often in mucosal cancer than in submucosal cancer (52.9\% vs $45.1 \%, P=0.003)$.

\section{Risk factors for LNM in mucosal gastric cancer and submucosal gastric cancer}

Univariate analysis indicated that age, sex, tumor size, LVI, differentiation type, and Lauren classification were risk factors for LNM (Table 4). The multivariate analysis showed that female sex, large tumor size, undifferentiatedtype, and LVI were independent risk factors for LNM (Table 5). Univariate analysis indicated that age, sex, tumor size, LVI, perineural invasion, differentiation-type, and Lauren classification showed an association with LNM in patients with submucosal gastric cancer (Table
4). The multivariate analysis indicated that female sex, younger age, LVI, and perineural invasion were the independent risk factors for LNM (Table 6). Female sex and LVI were both independent risk factors for LNM in mucosal and submucosal gastric cancer.

\section{Discussion}

EGC is more commonly diagnosed in Japan, where EGC accounts approximately $60 \%$ for all patients diagnosed as gastric cancer [2]. By contrast, the proportion of EGC in China varies from $10 \%$ to $20 \%$. Such great discrepancy

Table 5 Multivariate analysis of risk factors for lymph node metastasis in mucosal gastric cancer

\begin{tabular}{llll}
\hline Variables & Odds ratio & 95\% confidence interval & $P$ \\
\hline Age $(>60)$ & 0.981 & $0.953-1.01$ & 0.205 \\
Male sex & 0.341 & $0.163-0.715$ & 0.004 \\
Tumor size $(>2 \mathrm{~cm})$ & 1.38 & $1.07-1.779$ & 0.013 \\
Undifferentiated-type & 6.873 & $1.964-24.05$ & 0.003 \\
Lymphovascular invasion & 11.156 & $3.088-40.307$ & $<0.001$ \\
\hline
\end{tabular}


Table 6 Multivariate analysis of risk factors for lymph node metastasis in submucosal gastric cancer

\begin{tabular}{llll}
\hline Variables & Odds ratio & 95\% confidence interval & $P$ \\
\hline Age $(>60)$ & 0.976 & $0.956-0.997$ & 0.026 \\
Male sex & 0.557 & $0.354-0.875$ & 0.011 \\
Tumor size $(>2 \mathrm{~cm})$ & 1.2 & $0.993-1.45$ & 0.059 \\
Undifferentiated-type & 1.582 & $0.976-2.565$ & 0.062 \\
Lymphovascular invasion & 4.891 & $2.907-8.229$ & $<0.001$ \\
Perineural invasion & 3.673 & $1.62-8.325$ & 0.002
\end{tabular}

might be partly but not fully interpreted by the fact of more screening programs and different interpretations of pre-cancerous lesions in Japan, which furtherly has a large influence on the results of those studies analyzing risk factors for LNM in EGC. In Japan, regardless of the presence of invasive findings, not only structural atypia but also cellular atypia is sufficient to make a diagnosis of gastric cancer [14, 15]. However, these lesions could be diagnosed instead as high-grade dysplasia in our institution. This difference might result in a relatively higher incidence of EGC, a corresponding superior prognosis, and a lower incidence of LNM in patients with EGC in the Japanese cohort. Another reason might be the different efforts in pathological evaluation for early gastric cancer. For minority cases with conventional HE diagnosis of lymph node metastasis, immunohistochemistry will be added for further diagnosis. The thickness of paraffin sections is generally $4-6 \mu \mathrm{m}$. Thin slice specimen is not a routine in our center. Thus, the pathological staging of gastric cancer may be underestimated, resulting in a high incidence of lymph node metastasis rate. In addition, the worldwidely accepted indications of ER for EGC are mainly based on the clinical trials for Japanese patients and might not be exactly applicable to the patients in our country. It is important to define those patients benefiting from ER without compromising longterm survival or those patients being at high risk of LNM to receive curative gastrectomy instead of ER. Thus, the present study enrolled a relatively large number of EGC patients in a single institution from China and retrospectively investigated the incidence and risk factors for LNM in a Chinese population.

The status of LNM is the major concern in deciding the optimal treatment modality for EGC $[16,17]$. In the present study, LNM occurred in $16.7 \%$ of all EGC patients, with $8.7 \%$ in mucosal and $24.6 \%$ in submucosal gastric cancer, respectively. Our study found a relatively high LNM incidence in patients with EGC, which is notably higher than that of the Japanese cohort $[2,18]$. Despite the different rates of LNM, analyses of risk factors for LNM revealed a similar finding with those reported in the literature. Several studies had suggested large tumor size, ulcerative findings, submucosal invasion,
LVI, and undifferentiated-type cancer to be risk factors for LNM in EGC and recommended gastrectomy with LN dissection as the treatment option for those patients at high risk of LNM [19-21]. The present study also indicated that age, sex, tumor size, invasion depth, type of differentiation, LVI, and perineural invasion had significant effects on LNM in EGC by univariate and multivariate analyses. Depth of invasion is one of the most important risk factors for LNM. In our study, we compared clinicopathologic characteristics of mucosal cancer with submucosal cancer and did a subgroup analysis of risk factors in the mucosal cancer group and submucosal cancer group, respectively. Patients with submucosal gastric cancer had an older age, a higher proportion of upper lesion, larger tumor size, more frequent lymphovascular invasion and perineural invasion, and more LNM than patients with mucosal gastric cancer. Female sex and LVI were found to be the common risk factors in the two subgroups.

Majority of studies reported that more aggressive biological behavior and higher risk of metastasis were more frequently showed in young gastric cancer patients. Takatsu et al. [22] reported that younger patients are more likely to present with LNM, indicating that age was an independent risk factor. Another study by Lee et al. [23] reported that old age was associated with a lower risk for LNM in patients with EGC. This might result partly from a lower proportion of differentiated-type cancer in younger patients with EGC. However, other studies reported that there was no significant difference in LNM incidence or overall survival between younger and older patients [24]. In the present study, young age was demonstrated to be an independent risk factor for LNM in EGC patients. In the subgroup analysis, age also had an independent association with LNM in patients with submucosal gastric cancer.

Our study also indicated that female sex was an independent risk factor for LNM in EGC patients. The incidence of LNM was statistically higher in female patients than male patients. Other researchers also have reported that female sex is associated with LNM in EGC $[25,26]$. In female patients, the biological behavior of gastric cancer might be more aggressive, which could not be fully defined by tumor size, depth of invasion, or LVI [27]. Further studies were needed to investigate the biological association between sex and LNM.

In agreement with previously reported studies, our study also demonstrated that the tumor size, depth of invasion, and LVI are statistically significant risk factors for LNM in EGC. A previous study reported that no LNM was detected in mucosal cancers less than $2 \mathrm{~cm}$, indicating tumor size as an independent risk factor for LNM [28]. Generally, the depth of tumor invasion is tightly related with LNM in EGC. Both univariate and 
multivariate analyses in the present study demonstrated that a larger size tumor, submucosal invasion, and the presence of LVI and perineural invasion were significant predictive factors for LNM in patients with EGC. Among these risk factors, LVI might be the most important risk factor for LNM. In the present study, LVI was associated with LNM in both mucosal and submucosal lesions. Therefore, EGC with LVI is associated with a higher frequency of LNM, and surgical resection is necessary for EGC patients at high risk of LNM.

In our study, the rate of LNM in undifferentiated-type EGC was obviously higher than in differentiated-type. Undifferentiated-type was an independent risk factor for LNM in EGC. The more aggressive features of undifferentiated-type than the differentiated-type, have already been demonstrated [29]. Although ER has been indicated for undifferentiated EGC according to the expanded criteria, ER has generally been limited to differentiated-type EGC because of the higher risk of LNM in undifferentiated EGC. In the subgroup analyses, the present study also confirmed that undifferentiatedtype was an independent risk factor for LNM in mucosal cancer, whereas it was not in submucosal gastric cancer. Among these risk factors, LVI might be a more important factor for LNM in submucosal gastric cancer. In the present study, LVI was significantly associated with LNM in both mucosal and submucosal EGC.

We would indicate the criteria for endoscopic resection as those male patients with a differentiated-type EGC, of which the depth of invasion is clinically diagnosed as T1a, no ulcerative finding exists, no lymphovascular invasion, and the tumor size is no more than 2.0 $\mathrm{cm}$. Of 157 patients who met the above criteria in our study, no one has lymph node metastasis. Endoscopic resection should be considered for tumors that have a very low possibility of lymph node metastasis and are suitable for en bloc resection. According to the Japanese guidelines, tumors of the following categories have a very low possibility of lymph node metastasis when they are not accompanied with lymphovascular infiltration and could be indicated for endoscopic resection (expanded indication): of differentiated-type, ulcer (-), but $>2 \mathrm{~cm}$ in diameter; of differentiated-type, ulcer $(+)$, and $<3$ in diameter; and of undifferentiated-type, ulcer $(-)$, and < $2 \mathrm{~cm}$ in diameter. However, our research indicated that submucosal gastric cancer has a high incidence of lymph node metastasis. We do not recommend ER for submucosal gastric cancer. Nowadays, different gastric cancer treatment guidelines have different indications for endoscopic treatment of early gastric cancer. But the general principle should be the same, and only patients with a very low risk of lymph node metastasis are suitable for endoscopic treatment. Patients at high risk for lymph node metastasis should still undergo standard surgical resection and lymph node dissection. Our results show that the rate of lymph node metastasis in early gastric cancer in China is relatively high. If the endoscopic treatment guideline of Japanese and Korean is completely applied, a large part of patients with a higher risk of lymph node metastasis would receive endoscopic resection. Endoscopic treatment alone cannot achieve a curative resection for those patients, greatly increasing the possibility of recurrence and distant metastasis after endoscopic resection. Therefore, we must carefully choose the indications for endoscopic treatment. We cannot arbitrarily expand the indications for endoscopic treatment and blindly exaggerate the effect of endoscopic treatment. The overall long-term survival rate of patients remains the main therapeutic goal pursued.

Although the present study is one of the largest retrospective studies for EGC from a single institution in China, there are still some limitations. First, it was a retrospective study based on medical records in a single center. Second, only patients who underwent surgery were included in this study. Thus, possible selection bias was unavoidable. Third, our results are based on pathologic findings and not on clinical and endoscopic findings. Moreover, the depth of submucosal invasion is not specified (SM1, SM2, or SM3) in the present study. Therefore, our study might be not powerful enough to confirm all these related issues. To successfully utilize the endoscopic resection in China, criteria need to be strictly applied to the appropriate population, and further research including prospective, randomized, and controlled studies should be conducted.

\section{Conclusions}

In conclusion, our study revealed a relatively high incidence of LNM in Chinese patients with EGC. Sex, age, tumor size, depth of invasion, differentiation type, and lymphovascular and perineural invasion were identified as independent risk factors for LNM in EGC. LNM should be considered when deciding on the management of EGC. Minimal invasive treatment, such as ER, may be acceptable as a curative treatment in highly selective patients. Radical gastrectomy with LN dissection should be performed in EGC patients with a high risk of LNM in a Chinese population.

\section{Acknowledgements \\ Not applicable}

\section{Authors' contributions}

JC and YW built the conception and designed the study. JC and GZ provided statistical analysis and interpretation. JC wrote, reviewed, and revised the manuscript. The authors read and approved the final manuscript.

Funding

This study was performed without funding. 


\section{Availability of data and materials}

The datasets generated and/or analyzed during the current study are not publicly available because they are derived from the patient database of the center and hence subject to confidentiality but are available from the corresponding author on reasonable request.

\section{Ethics approval and consent to participate}

The ethical committee of Fudan University Shanghai Cancer Center approved this study. Because of the retrospective nature of the study, the requirement for informed consent was waived.

\section{Consent for publication}

Not applicable

\section{Competing interests}

The authors declare that they have no competing interests

Received: 20 October 2019 Accepted: 17 March 2020

Published online: 20 March 2020

\section{References}

1. Kajitani T. The general rules for the gastric cancer study in surgery and pathology. Part I. Clinical classification. Jpn J Surg. 1981;11:127-39.

2. Gotoda T, Yanagisawa A, Sasako M, et al. Incidence of lymph node metastasis from early gastric cancer: estimation with a large number of cases at two large centers. Gastric Cancer. 2000;3:219-25.

3. Choi AH, Nelson RA, Merchant SJ, et al. Rates of lymph node metastasis and survival in T1a gastric adenocarcinoma in western populations. Gastrointest Endosc. 2016;83(6):1184-92.

4. Noh SH, Hyung WJ, Cheong JH. Minimally invasive treatment for gastric cancer: approaches and selection process. J Surg Oncol. 2005;90:188-93.

5. Kwee RM, Kwee TC. Predicting lymph node status in early gastric cancer Gastric Cancer. 2008;11:134-48.

6. Lee KS, Oh DK, Han MA, et al. Gastric cancer screening in Korea: report on the national cancer screening program in 2008. Cancer Res Treat. 2011;43: 83-8.

7. Pelz J, Merkel S, Horbach T, et al. Determination of nodal status and treatment in early gastric cancer. Eur J Surg Oncol. 2004;30:935-41.

8. Borie F, Millat B, Fingerhut A, et al. Lymphatic involvement in early gastric cancer: prevalence and prognosis in France. Arch Surg. 2000;135:1218-23.

9. Roviello F, Rossi S, Marrelli D, et al. Number of lymph node metastases and its prognostic significance in early gastric cancer: a multicenter Italian study. J Surg Oncol. 2006;94:275-80.

10. Ono $\mathrm{H}$, Kondo $\mathrm{H}$, Gotoda $\mathrm{T}$, et al. Endoscopic mucosal resection for treatment of early gastric cancer. Gut. 2001;48:225-9.

11. Pyo $\mathrm{JH}$, Lee $\mathrm{H}, \mathrm{Min} \mathrm{BH}$, et al. Long-term outcome of endoscopic resection vs. surgery for early gastric cancer: a non-inferiority- matched cohort study. Am J Gastroenterol. 2016;111:240-9.

12. Kodera Y, Sano T. Japanese gastric cancer treatment guidelines (JGCTG) version 4, 2014. Gastric Cancer. 2017;20:1-19.

13. Chen W, Zheng R, Baade PD, et al. Cancer statistics in China, 2015. CA Cancer J Clin. 2016;66:115-32.

14. Schlemper RJ, Itabashi M, Kato Y, et al. Differences in diagnostic criteria for gastric carcinoma between Japanese and Western pathologists. Lancet. 1997;349:1725-9.

15. Schlemper RJ, Kato Y, Stolte M. Review of histological classifications of gastrointestinal epithelial neoplasia: differences in diagnosis of early carcinomas between Japanese and Western pathologists. J Gastroenterol. 2001;36:445-56

16. Gotoda T. Endoscopic resection of early gastric cancer. Gastric Cancer. 2007; 10:1-11.

17. Kang KJ, Kim KM, Min BH, et al. Endoscopic submucosal dissection of early gastric cancer. Gut Liver. 2011;5:418-26.

18. Sekiguchi M, Oda I, Taniguchi H, et al. Risk stratification and predictive riskscoring model for lymph node metastasis in early gastric cancer. J Gastroenterol. 2016;51:961-70.

19. Fang $W L$, Huang $K H$, Lan $Y T$, et al. The risk factors of lymph node metastasis in early gastric cancer. Pathol Oncol Res. 2015;21:941-6.

20. Ren G, Cai R, Zhang WJ, et al. Prediction of risk factors for lymph node metastasis in early gastric cancer. World J Gastroenterol. 2013;19:3096-107.
21. Kanesaka T, Nagahama T, Uedo N, et al. Clinical predictors of histologic type of gastric cancer. Gastrointest Endosc. 2018;87:1014-22.

22. Takatsu Y, Hiki N, Nunobe $S$, et al. Clinicopathological features of gastric cancer in young patients. Gastric Cancer. 2016;19:472-8.

23. Lee $\mathrm{JH}, \mathrm{Nam} \mathrm{BH}$, Ryu KW, et al. Tumor differentiation is not a risk factor for lymph node metastasis in elderly patients with early gastric cancer. Eur J Surg Oncol. 2014;40:1771-6.

24. Hsieh FJ, Wang YC, Hsu JT, et al. Clinicopathological features and prognostic factors of gastric cancer patients aged 40 years or younger. J Surg Oncol. 2012;105:304-9.

25. Yasuda K, Shiraishi N, Suematsu T, et al. Rate of detection of lymph node metastasis is correlated with the depth of submucosal invasion in early stage gastric carcinoma. Cancer. 1999;85:2119-23.

26. Abe N, Sugiyama M, Masaki T, et al. Predictive factors for lymph node metastasis of differentiated submucosally invasive gastric cancer. Gastrointest Endosc. 2004;60:242-5.

27. Abe N, Watanabe T, Suzuki K, Machida H, et al. Risk factors predictive of lymph node metastasis in depressed early gastric cancer. Am J Surg. 2002; 183:168-72.

28. Hölscher AH, Drebber U, Mönig SP, et al. Early gastric cancer: lymph node metastasis starts with deep mucosal infiltration. Ann Surg. 2009;250:791-7.

29. Oh SY, Lee KG, Suh YS, et al. Lymph node metastasis in mucosal gastric cancer: reappraisal of expanded indication of endoscopic submucosal dissection. Ann Surg. 2017;265:137-42.

\section{Publisher's Note}

Springer Nature remains neutral with regard to jurisdictional claims in published maps and institutional affiliations.
Ready to submit your research? Choose BMC and benefit from:

- fast, convenient online submission

- thorough peer review by experienced researchers in your field

- rapid publication on acceptance

- support for research data, including large and complex data types

- gold Open Access which fosters wider collaboration and increased citations

- maximum visibility for your research: over $100 \mathrm{M}$ website views per year

At $\mathrm{BMC}$, research is always in progress.

Learn more biomedcentral.com/submissions 